\title{
SEXO, MENTIRAS E INTERNET
}

\author{
Rafael Ballester-Arnal \\ rballest@uji.es \\ Jesús Castro-Calvo \\ Estefanía Ruiz-Palomino \\ Beatriz Gil-Juliá \\ Cristina Giménez-García
}

Salusex-Unisexsida. Dpto. Psicología Básica, Clínica y Psicobiología.

Universitat Jaume I de Castellón (Spain)

Fecha de Recepción: 6 Febrero 2018

Fecha de Admisión: 10 Abril 2018

\section{RESUMEN}

Cada vez más utilizamos internet para relacionarnos socialmente y también para entablar relaciones sexuales. El cibersexo puede aportar algunos beneficios, pero también facilita la exposición a riesgos como la facilidad con la que se puede mentir acerca de la propia identidad y características y la dificultad de contrastar esta información por parte del interlocutor. La motivación puede ser explorar la propia sexualidad, pero también intentar ocultar complejos o el intento de engañar a la otra persona. Nuestro objetivo es analizar hasta qué punto las mentiras son frecuentes en el cibersexo y su relación con diferentes variables. Administramos a 1907 personas (45,9\% mujeres) entre 18-50 años (Media=27,9, DT=9,72) una batería online. Un 97,4\% de hombres y 75,8\% de mujeres dijeron haber utilizado internet con fines sexuales (Chi cuadrado=141,89, $p<0,000$ ). Un 31,3\% de esos hombres dijeron haber mentido sobre algún aspecto de su persona, frente a un $24,5 \%$ de las mujeres (Chi cuadrado=5,36, $p<0,021$ ). El contenido de las mentiras fue principalmente aspectos de su vida (estudios, trabajo, familia, amigos...) con el 10,8\% de los hombres y el 7,4\% de las mujeres; seguido de la edad (10,6\% y 6,5\% respectivamente); el aspecto físico (7,7\% y 4,6\%); y el hecho de ser hombre o mujer (3,2\% y 1,8\%). No se apreció relación con la edad, ni la autoestima, pero sí con el número de horas conectado a páginas sexuales, la preocupación acerca del uso del cibersexo, el grado de compulsividad sexual, la adicción al cibersexo, al sexo y a internet y la búsqueda de sensaciones sexuales. Mientras que el porcentaje de heterosexuales que habían mentido era del $21 \%$, en los bisexuales y homosexuales superaba el $40 \%$ (Chi cuadrado $=55,74, p<0,000$ ). En conclusión, las mentiras en cibersexo no sólo son frecuentes sino que guardan una relación estrecha con un uso problemático o adictivo del mismo.

Palabras clave: cibersexo; mentiras; diferencias de género; variables asociadas 


\section{ABSTRACT}

\section{Sex, lies and the internet.}

The Internet more increasingly seems to be used to socialize as well as to engage in sexual relations. Cybersex can provide some benefits, but also facilitates the exposure to some risks such as the easiness of lying about one's identity and characteristics, and the difficulty of verifying this information by the interlocutor. The motivation may be to explore one's sexuality, but also try to hide complexes or try to deceive the other person. Our objective is to analyse to what extent lies are frequent in cybersex and their relation to different variables. 1.907 people (45.9\% women) aged between 18-50 years (Mean=27.9; $\mathrm{SD}=9.72$ ) were administered an online battery. $97.4 \%$ of men and $75.8 \%$ of women used the Internet for sexual purposes (Chi square $=141.89, p<0.000$ ). $31.3 \%$ of these men lied about some aspects of their appearance, compared to $24.5 \%$ of women (Chi square $=5.36, p<0.021$ ). The content of the lies was mainly different aspects of his/her life (studies, work, family, friends, ...) with $10.8 \%$ of men and $7.4 \%$ of women; followed by age $(10.6 \%$ and $6.5 \%$ respectively); the physical appearance $(7.7 \%$ and $4.6 \%)$; and the fact of being man or woman $(3.2 \%$ and $1.8 \%$ ). There was no relationship with age and self-esteem, but was with the number of hours connected to sexual websites, the concern about the cybersex use, the degree of sexual compulsivity, addiction to cybersex, sex and the internet, and sexual sensation seeking. While the percentage of heterosexuals who lied was $21 \%$, in bisexuals and homosexuals it exceeded $40 \%$ (Chi square $=55.74, p<0.000)$. In conclusion, the lies in cybersex are not only frequent but also closely related to a problematic or addictive use of it.

Keywords: cybersex; lies; gender differences; associated variables

\section{Este trabajo ha sido financiado por el proyecto P1.1B2015-82 (Universitat Jaume I)}

\section{ANTECEDENTES}

El uso de internet se ha extendido en nuestras vidas hasta tal punto que resulta difícil ya imaginarnos el día a día sin él. Internet cubre muchas de nuestras necesidades: reservamos viajes, compramos billetes de avión, buscamos direcciones, compramos libros, trabajamos bajo la opresión de miles de correos electronicos, nos hacemos nuestras páginas de presentación donde colgamos las fotos y las actividades que hacemos para que todo el mundo las conozca y estar hiperconectados con el universo, establecemos contacto con otras personas y también en ocasiones buscamos satisfacer nuestras necesidades sexuales a través de internet.

Técnicamente definimos el cibersexo, como "el uso de Internet con objetivos de gratificación sexual", pero detrás de esta definición nos encontramos con muchísimas actividades diferentes que el ser humano puede realizar. Algunas son solitarias como el visionado de imágenes o películas pornográficas. Otras implican una interacción con otras personas, intercambiando imágenes como en el sexting, manteniendo relaciones que incluyen el visionado mutuo a través de la webcam, o manteniendo conversaciones de carácter erótico a través de los chats.

Todas estas actividades tienen una prevalencia mayor de lo que cabría imaginar. Según un estudio realizado por nuestro equipo con población adolescente (Ballester-Arnal, Giménez-García, GilLLario \& Castro-Calvo, 2016), un 49,2\% de chicos busca material sexual a través de internet, frente al $6,7 \%$ de las chicas; un $60,6 \%$ de chicos se ha masturbado mientras estaba conectado comparado con el 7,3\% de las chicas; un $6,5 \%$ de los chicos ha tenido contactos en persona con usuarios a los que había conocido en internet versus el $8,1 \%$ de las chicas; un 10,2\% de chicos ha participado en chats sexuales frente al $4 \%$ de las chicas; un $12,7 \%$ de los chicos reconoce que el cibersexo ha interferido en sus vidas comparado con el $4,7 \%$ de las chicas; y finalmente, un $6,4 \%$ de los chicos creía padecer una adicción versus el $0,7 \%$ de las chicas. 
Y en población joven, el estudio de Ballester-Arnal, Castro-Calvo, Gil-LLario y Gil-Juliá (2017), realizado con 1557 participantes, revela que un 35,5\% busca material sexual en internet, un 30,2\% se ha masturbado mientras estaba conectados, un $24,8 \%$ ha mantenido contactos sexuales con otras personas, un $17,7 \%$ ha tenido relaciones sexuales en persona con usuarios a los que habían conocido a través de internet y un 12,9\% había participado en chats sexuales. Un 7,4\% manifestaba que el cibersexo interfería con sus vidas y un $4 \%$, creía padecer una adicción. De nuevo, en población joven existen grandes diferencias entre hombres y mujeres. Por ejemplo, el porcentaje de los que se masturban mientras están conectados a internet es del $59 \%$ en chicos frente al $14,3 \%$ en chicas; 0 el de los que se han conectado a chats sexuales es del 20,5\% de chicos frente al $8,6 \%$ de las chicas.

Estos datos no difieren demasiado al de otros estudios internacionales como el de Daneback, Cooper y Månsson (2005), Shaughnessy, Byers y Walsh (2011) o Wéry y Billieux (2017).

Existe una cierta controversia acerca de cuáles pueden ser los efectos beneficiosos y negativos del consumo de cibersexo. Independientemente de posturas que pueden tener más de ideología que de rigor científico, y sin ánimo de ser exhaustivos, las evidencias nos dicen que Internet puede ser una herramienta positiva para satisfacer los impulsos sexuales (Daneback, Sevcikova, Mänsson, \& Ross, 2013) o de encontrar parejas en un entorno bastante seguro (Courtice \& Shaughnessy, 2018), algo que podría ser especialmente importante en personas con una orientación sexual estigmatizada. Sin embargo, también es cierto que existen ciertos riesgos asociados al cibersexo, no solo por lo que se refiere a la posibilidad de caer en un uso excesivo y sin control que acabe interfiriendo en distintos ámbitos de la vida, sino por otros motivos, como el desarrollo de mitos y prejuicios alejados de la realidad o la exposición a riesgos como los derivados de compartir información demasiado íntima con personas a las que no se conoce de nada o que pueden utilizarla de manera indeseable.

Efectivamente, Internet puede ser un entorno ideal para el desarrollo de las fantasías sexuales. El modo de fantasear puede implicar una actitud más pasiva y solitaria (p.e, visualizar escenas que representan prácticas sexuales que a uno le gustaría hacer imaginando que nos encontramos en esa situación); o más activa y social, implicando al propio yo, como la construcción de un avatar que representa a la propia persona interactuando con otros personajes que tampoco son reales (como en las aplicaciones Second Life); o con una implicación de la persona real que interactúa con otra persona real 0 bien a través de la webcam o sobre todo, del chat. Es en este contexto donde las fantasías que tan importantes y positivas son para la vida sexual de cualquier persona, pueden convertirse fácilmente en mentiras más arriesgadas. Internet facilita construirse una identidad que nada tenga que ver con la realidad. Aunque la relación sea a través de webcam, el usuario puede dar información falsa acerca de su vida como en qué trabaja o su situación personal, difícil de contrastar. Pero sobre todo, si es a través de chat, resulta particularmente fácil mentir acerca de la edad, el sexo o el aspecto físico.

Las fantasías o mentiras no tienen por qué ser peligrosas. Pueden ser comprensibles cuando pretenden ocultar algún complejo sexual o físico. A veces ayudan a autoconocerse explorando otras identidades sexuales en un entorno seguro. Incluso pueden ser divertidas cuando son compartidas con el otro. Pero en ocasiones pueden acabar convirtiéndose en un engaño continuado a uno mismo, perdiendo el sentido de la realidad en una doble vida; 0 a otras personas, con el fin de utilizarlas, engañarlas o incluso acosarlas, como ocurre en el fenómeno del grooming.

Todos estos aspectos han sido muy poco investigados incluso dentro de la literatura sobre cibersexo que se inició hace apenas casi dos décadas y desde luego, no hay un solo estudio en nuestro país al respecto. De este vacío surge el presente trabajo. 


\section{OBJETIVO}

El objetivo que perseguimos con este trabajo es analizar hasta qué punto las mentiras son frecuentes en la práctica del cibersexo. Como objetivos secundarios, se intenta averiguar el tipo de mentiras más frecuentes, la existencia de diferencias en función del género y la orientación sexual y la relación que tiene el comportamiento de mentir durante las interacciones sexuales a través de internet, con otras variables como el grado de uso y abuso de internet, de cibersexo, el grado de control de los impulsos sexuales (compulsividad sexual), la hipersexualidad y adicción al sexo, la búsqueda de sensaciones sexuales y la autoestima.

\section{PARTICIPANTES}

El número de participantes fue de 1907 personas, de los cuales el 45,9\% eran mujeres y el resto hombres. El rango de edad estuvo comprendido entre 18-50 años, con una media de 27,9 años (DT=9,72). Por orientación sexual, el $71 \%$ se declaraba heterosexual, el 13,6\% como bisexual y el $15,4 \%$ como homosexual.

\section{METODOLOGÍA E INSTRUMENTOS UTILIZADOS}

Los datos utilizados para este trabajo forman parte de un estudio nacional en el que se recogió muestra a partir de un link asociado a una batería online. Los participantes fueron reclutados a través de anuncios colocados en Facebook en los que se exponía el link por el que podían acceder a la plataforma ADISEX que creamos para tal fin (https://adiccionalsexo.uji.es/). Tras obtener su consentimiento informado, una vez cumplimentados los cuestionarios, la plataforma les devolvía de forma anónima un informe automático de los resultados referidos a su conducta relacionada con el cibersexo. Los instrumentos de evaluación cuyos resultados han sido utilizados para este trabajo son los siguientes:

Cuestionario ad-hoc. Recoge información de variables sociodemográficas, comportamiento relacionado con el uso de internet (horas conectado/a a internet), conducta sexual en general (p.e. orientación sexual) y uso del ordenador con fines sexuales. Respecto a las variables asociadas al uso de internet con fines sexuales utilizadas en este trabajo se evaluó si se había utilizado internet con fines sexuales, número de horas conectado a páginas sexuales, si se ha mentido alguna vez en las interacciones con otras personas a través del cibersexo y el aspecto sobre el que han versado tales mentiras.

Internet Sex Screening Test (ISST) de Delmonico (1997). Adaptación española de Ballester, Gil, Gómez y Gil (2010). La versión original incluye 25 ítems de verdadero/falso y mide la conducta sexual online. Validado el instrumento original con población general (a través de la autoadministración online), el análisis factorial exploratorio reveló cinco factores (Delmonico \& Miller, 2003): Compulsividad Sexual Online, Conducta sexual social online, Conducta sexual solitaria; Gasto sexual online; e Interés en la conducta sexual online. Su fiabilidad osciló entre 0,51 y 0,86 . Aplicándolo a una muestra de 1239 jóvenes españoles se obtuvo la versión española de Ballester et al, con una consistencia interna de 0,88 y una estabilidad test-retest de 0,84.

El Cuestionario de Screening de Adicción al Sexo Revisado (SAST-R) de Carnes, Green y Carnes (2010) que consta de 45 items de respuesta dicotómica. Evalúa cuatro dimensiones: Preocupación, Pérdida de control, Problemas de relación y Trastorno afectivo. Su fiabilidad para distintas muestras se sitúa entre 0.812 y 0.919 . Se utiliza la versión traducida y validada por nuestro grupo de investigación (Castro-Calvo, Ballester-Arnal, Billieux, Gil-Juliá \& Gil-Llario, en revision).

Cuestionario de Adicción a Internet (CAI) de Young (2003), con 20 ítems tipo Likert (raramente/ocasionalmente/frecuentemente/a menudo/siempre). En su versión original, autoadministrada online, tiene seis factores y las subescalas han mostrado una consistencia interna alfa de Cronbach 
entre 0,54 y 0,82 (Widyanto \& McMurran, 2004). Su fiabilidad (consistencia interna) en muestra española de jóvenes universitarios es de 0,910 (Salmerón, Ballester, Gómez \& Gil, 2009).

Escala revisada de Búsqueda de sensaciones sexuales de Kalichman y Rompa (1995). Evalúa uno de los constructos más relacionados con la compulsividad sexual y las conductas sexuales de riesgo, la búsqueda de sensaciones sexuales. Consta de 11 items que se responden siguiendo una escala Likert de 4 puntos (nada a muy de acuerdo). Consistencia interna de 0,87. Se utilizó la traducción validada realizada por nuestro grupo de investigación (Ballester-Arnal, Ruiz-Palomino, Espada-Sánchez, Morell-Mengual \& Gil-Llario, 2018).

El SCS (Sexual Compulsivity Scale de Kalichman) (Kalichman \& Rompa, 1995) en la versión adaptada y validada para su aplicación en nuestro país por nuestro equipo (Ballester, Gómez, Gil \& Salmerón, 2012). Consistencia interna de 0.84 .

RSEl. Cuestionario de autoestima de Rosenberg (1979). Este cuestionario contiene 10 ítems y proporciona información cualitativa y cuantitativa sobre la autoestima. Los elevados niveles de validez y fiabilidad de esta escala (Alpha de Cronbach de 0,87 y fiabilidad test-retest de 0,85 ) han sido ampliamente documentados.

Hypersexual Behavior Inventory (HBI) (Reid, Garos \& Carpenter, 2011). Se trata de una escala de 19 items tipo Likert diseñada para medir las tres dimensiones básicas de la hipersexualidad: Afrontamiento (uso del sexo para responder a estados de ánimo disfóricos), Control (problemas para controlar y reducir los pensamientos, impulsos y conductas sexuales) y Consecuencias (persistencia de las fantasias, impulsos y conductas a pesar de las consecuencias negativas). La fiabilidad obtenida por los autores para las distintas escalas oscila entre 0,89 y 0,95 . Para este trabajo se utilizó la traducción y validación realizada por nuestro equipo (Ballester-Arnal, Gil-Llario, CastroCalvo \& Gil-Juliá, en revisión).

\section{ANÁLISIS ESTADÍSTICOS}

Se utilizó el programa SPSS para la realización de los análisis estadísticos. Además de los estadísticos descriptivos para las variables relacionadas con el uso del cibersexo, se realizaron pruebas chi cuadrado para el análisis de diferencias de género y en función de la orientación sexual y correlaciones de Spearman para el cálculo de las relaciones entre variables.

\section{RESULTADOS}

Un $97,4 \%$ de hombres y $75,8 \%$ de mujeres dijo haber utilizado internet con fines sexuales (Chi cuadrado $=141,89, p<0,000)$.

Un $31,3 \%$ de los hombres que afirmaron haber mantenido contactos sexuales online con otros usuarios a través de chat, videoconferencia... dijeron haber mentido sobre algún aspecto de su persona, frente a un $24,5 \%$ de las mujeres (Chi cuadrado=5,36, $p<0,021$ ).

Como se puede apreciar en la tabla 1, el contenido de las mentiras fue principalmente aspectos de su vida (estudios, trabajo, familia, amigos...) con el 10,8\% de los hombres y el 7,4\% de las mujeres de la muestra total; seguido de la edad (10,6\% y 6,5\% respectivamente); el aspecto físico (7,7\% y $4,6 \%)$; y el hecho de ser hombre o mujer (3,2\% y $1,8 \%$ ).

Si el análisis lo hacemos respecto a los que afirman haber mentido, los porcentajes pasan a ser del $56,6 \%$ de los hombres y $64,2 \%$ de las mujeres que han mentido sobre diferentes aspectos de su vida; el $56,6 \%$ y $57,9 \%$ respectivamente que han mentido sobre su edad; el $41,8 \%$ y $40 \%$ respectivamente que han mentido sobre su aspecto físico, y el $17,5 \%$ y $16,8 \%$ respectivamente que han mentido sobre el hecho de ser hombres o mujeres.

Las diferencias entre hombres y mujeres fueron estadísticamente significativas en las mentiras sobre el aspecto físico (Chi cuadrado=7,75, $p<0,005$ ), la edad (Chi cuadrado=9,84, $p<0,002$ ), y otros 
aspectos de su vida (Chi cuadrado=6,32, $p<0,012$ ), siendo los hombres los que mienten en mayor medida

Tabla 1. Diferencias de género en cuanto al tipo de mentiras en cibersexo en la muestra total

\begin{tabular}{lcccc}
\hline \multicolumn{1}{c}{ Tipo de mentira } & $\begin{array}{c}\text { Hombres } \\
(\%)\end{array}$ & $\begin{array}{c}\text { Mujeres } \\
(\%)\end{array}$ & Chi $^{2}$ & $\mathrm{p}$ \\
\hline El hecho de ser hombre o mujer & 3,2 & 1,8 & 3,571 & 0,059 \\
El aspecto físico & 7,7 & 4,6 & 7,75 & 0,005 \\
La edad & 10,6 & 6,5 & 9,84 & 0,002 \\
Aspectos de su vida (estudios, trabajo, & 10,8 & 7,4 & 6,32 & 0,012 \\
familia, renta...) & 1,4 & 0,8 & 20,15 & 0,512 \\
Otros & & & & \\
\hline
\end{tabular}

En cuanto a la relación entre los distintos tipos de mentiras, como se puede apreciar en la tabla 2 , todas correlacionan significativamente entre sí $(p<0,000)$ lo que parece indicar un cierto patrón generalizado de manera que los que suelen mentir sobre por ejemplo la edad, también lo hacen sobre el hecho de ser hombre o mujer, sobre su condición física o sobre otros aspectos de su vida.

Tabla 2. Relación entre los distintos tipos de mentiras en cibersexo (correlaciones de Spearman)

\begin{tabular}{lllll}
\hline & $\begin{array}{c}\text { Haber } \\
\text { mentido } \\
\text { alguna vez }\end{array}$ & $\begin{array}{c}\text { El hecho de } \\
\text { ser hombre } \\
\text { o mujer }\end{array}$ & $\begin{array}{c}\text { Aspecto } \\
\text { físico }\end{array}$ & Edad \\
\hline Haber mentido alguna vez & & & & \\
\hline El hecho de ser hombre o mujer & $\begin{array}{l}\text { rho=0,360 } \\
\mathrm{p}<0,000\end{array}$ & & & \\
& $\mathrm{rho}=0,577$ & $\mathrm{rho}=0,314$ & & \\
& $\mathrm{p}<0,000$ & $\mathrm{p}<0,000$ & & \\
\hline El aspecto físico & $\mathrm{rho}=0,687$ & $\mathrm{rho}=0,326$ & $\mathrm{rho}=0,420$ & \\
& $\mathrm{p}<0,000$ & $\mathrm{p}<0,000$ & $\mathrm{p}<0,000$ & \\
\hline La edad & $\mathrm{rho}=0,696$ & $\mathrm{rho}=0,326$ & $\mathrm{rho}=0,41$ & $\mathrm{rho}=0,519$ \\
& $\mathrm{p}<0,000$ & $\mathrm{p}<0,000$ & $\mathrm{p}<0,000$ & $\mathrm{p}<0,000$ \\
\hline Aspectos de su vida (estudios, & & & & \\
trabajo, familia, renta...) & & & & \\
\hline
\end{tabular}

Respecto a la relación entre el comportamiento de mentir en las interacciones sexuales en internet y otras variables, no se apreció ninguna correlación significativa con la edad (rho=-0,030, $p<0,345)$, ni la autoestima ( $r h 0=-0,052, p<0,158$ ), pero sí con el número de horas conectado a internet $(r h o=-0,113, p<0,000)$, a páginas sexuales ( $r h 0=-0,166, p<0,000)$, el grado de compulsividad sexual (rho $=-0,112, p<0,002$ ), la hipersexualidad ( $r h 0=-0,142, p<0,000$ ), la adicción al cibersexo (rho $=-0,333, p<0,000)$, al sexo $(r h o=-0,155, p<0,000)$ y a internet $(r h o=-0,093, p<0,011)$ y la búsqueda de sensaciones sexuales ( $r$ ho $=-0,120, p<0,001$ ).

Finalmente, realizamos análisis diferenciales en función de la orientación sexual. Como se puede apreciar en la tabla 3, de entrada, el uso de internet con fines sexuales fue significativamente mayor (Chi cuadrado $=41,55, p<0,000)$ en homosexuales $(98,6 \%)$ y bisexuales $(93,5 \%)$ que en heterosexuales $(83,8 \%)$. Mientras que el porcentaje de heterosexuales que habían mentido era del $21 \%$, en los bisexuales y sobre todo los homosexuales superaba el $40 \%$ (Chi cuadrado $=55,74, p<0,000$ ). Por lo que respecta al contenido de las mentiras, en todos los casos las diferencias entre las personas con diferentes orientaciones sexuales son estadísticamente significativas $(p<0,000)$ con porcentajes 
dos o tres veces inferiores en los heterosexuales comparados con el resto. El tipo de mentira más frecuente en todos ellos versa sobre la edad u otros aspectos como los estudios, trabajos, familia, renta..., seguido por el aspecto físico y finalmente por el hecho de ser hombre o mujer. De nuevo, en casi todos los casos, los homosexuales obtienen los mayores porcentajes excepto en el mentir sobre el hecho de ser hombre o mujer donde es mayor el porcentaje entre bisexuales que entre homosexuales.

Tabla 3. Análisis diferenciales en función de la orientación sexual

\begin{tabular}{lccccc}
\hline & Heterosex. & Bisex. & Homosex. & $\mathrm{Chi}^{2}$ & $\mathrm{p}$ \\
\hline Uso de Internet con fines sexuales & 83,8 & 93,5 & 98,6 & 41,55 & 0,000 \\
Alguna vez ha mentido & 20,8 & 40,4 & 45,3 & 55,74 & 0,000 \\
Mentir sobre ser hombre o mujer & 1,9 & 6,6 & 5,8 & 19,69 & 0,000 \\
Mentir sobre el físico & 5,2 & 11,7 & 14,9 & 32,67 & 0,000 \\
Mentir sobre la edad & 7 & 16 & 22 & 54,84 & 0,000 \\
Mentir sobre otros aspectos & 7,8 & 18,3 & 20,3 & 43,86 & 0,000 \\
\hline
\end{tabular}

\section{DISCUSIÓN Y CONCLUSIONES}

En el año 1989 se estrenó con enorme éxito la película estadounidense "Sex, Lies, and Videotape" dirigida magistralmente por Steven Soderbergh, que narraba la historia de un abogado, adicto al sexo, que tenía una aventura con la hermana de su mujer, absolutamente segura de su matrimonio, hasta que entraba en escena un antiguo compañero de su marido, que complicaba la trama.

Las mentiras forman parte del quehacer humano. Son frecuentísimas en todos nosotros y sin entrar en los casos patológicos en los que se aprecia una cierta compulsividad hacia el mentir, lo cierto es que a las personas nos encanta falsear la realidad. Las razones que nos empujan a mentir pueden ser muy variadas. A veces intentamos cubrir con ellas algún comportamiento del que no nos sentimos especialmente orgullosos. Otras veces nos son útiles para no dejar entrever la animadversion que nos causan ciertas personas y en ese sentido, resultan fundamentales para nuestra socialización y para no estar en guerra continua con nuestros congeneres. En ocasiones nos mentimos a nosotros mismos porque una parte de la realidad no nos gusta. Puede ser una característica de nuestra propia persona. Puede ser el diagnóstico de una enfermedad propia 0 de un ser querido que no podemos soportar emocionalmente. 0 cualquier situación que nos resistimos a aceptar.

En el ámbito de nuestra sexualidad quizás las mentiras tengan todavía mayor acto de presencia. Quizás tres aspectos expliquen esto. Por un lado, el hecho de que gran parte de nuestra sexualidad tiene un componente relacional, que nos conecta con personas cuya opinion nos importa y mucho. Por otro lado, el hecho de que la sexualidad en nuestra cultura está sobrevalorada, de manera que nos interesa creer y hacer saber que somos excelentes amantes, que tenemos una supedotación sexual expresada a través del tamaño del pene, la facilidad para experimentar excitaciones, la capacidad para tener multiples orgasmos en muy poco tiempo y para hacer estallar de placer a cualquier persona que se introduzca en nuestra cama. Todo ello, porque esa sobrevaloración hace que gran parte de nuestra autoestima resida en una parte demasiado pequeña de nuestra persona. $Y$ en tercer lugar, porque la sexualidad sigue siendo un tema tabú sobre el que han caído tantas restricciones que necesitamos disimular y mentir continuamente cuando nuestra sexualidad no se ajusta a lo que la sociedad espera de nosotros.

La práctica del cibersexo es cada vez es más frecuente en nuestra población. Las razones esgrimidas anteriormente, junto con las propias características del cibersexo, hacen que la posibilidad de 
mentir se multipliquen en este ámbito. Nuestro estudio ha revelado que un porcentaje importante de personas, especialmente los hombres, mienten mientras están conectados a internet, tanto sobre su aspecto físico y otros aspectos de su vida, como sobre su edad e incluso sobre su identidad sexual. Parece haber un cierto factor $g$ que hace que quien miente sobre un aspecto tienda a mentir sobre los otros. Por otro lado, esta tendencia es mayor en personas que tienen una orientación no heterosexual, seguramente por los imperativos y el impacto de una sociedad homófoba que impide a las personas no heterosexuales expresarse con libertad. Y guarda una relación con variables clínicas como la hipersexualidad, compulsividad sexual, la adicción a internet, al sexo y al cibersexo y con la búsqueda de sensaciones sexuales.

Sin embargo, necesitamos seguir investigando acerca de la tendencia humana a crear realidades alternativas en internet especialmente en el ámbito de la sexualidad. Sería muy intereante conocer las motivaciones para hacerlo, las características de personalidad asociadas y el impacto real que esas mentiras tienen sobre las propias personas que las crean. Y también sobre las personas con las que interactúan que pueden llegar a ser víctimas no solo de engaños o infidelidades sino de manipulaciones e incluso de distintas formas de acoso, especialmente peligrosas en el caso de los menores.

\section{REFERENCIAS BIBLIOGRÁFICAS}

Ballester-Arnal, R., Giménez-García, C., Gil-Llario, M.D., \& Castro-Calvo, J. (2016). Cybersex in the "Net generation": Online sexual activities among Spanish adolescents. Computers in Human Behavior, 57, 261-266.

Ballester-Arnal, R., Castro-Calvo, J., Gil-Llario, M.D. \& Gil-Juliá, B. (2017). Cybersex addiction: a study on Spanish college students. Journal of Sex \& Marital Therapy, 43, 567-585. D0I: 10.1080/0092623X.2016.1208700.

Ballester-Arnal, R., Gil-Llario, M.D., Castro-Calvo, J. \& Gil-Juliá, B. (en revisión). A validation study of the Spanish version of the Hypersexual Behavior Inventory (HBI): pencil and paper versus On line administration.

Ballester, R., Gil, MD., Gómez, S. \& Gil, B. (2010). Propiedades psicométricas de un instrumento para evaluar la adicción al cibersexo. Psicothema, 22, 1048-1053.

Ballester, R., Gómez, S., Gil, M.D. \& Salmerón, P. (2012). Sexual compulsivity scale (SCS): Adaptation and validation in Spanish population. Journal of Sex \& Marital Therapy, 6(39), 526540.

Ballester-Arnal, R., Ruiz-Palomino, E., Espada-Sánchez, J.P., Morell-Mengual, V. \& Gil-Llario, M.D. (2018). Psychometric properties and validation of the sexual sensation seeking scale in Spanish adolescents: Brief screening method for use in research and clinical practice. Personality and Individual Differences, 122, 47-54.

Carnes, P., Green, B. \& Carnes, S. (2010). The same yet different: refocusing the Sexual Addiction Screening Test (SAST) to reflect orientation and gender. Sexual Addiction and Compulsivity, 17, 1, 7-30.

Castro-Calvo, J., Ballester-Arnal, R., Billieux, J., Gil-Juliá, B. \& Gil-Llario, M.D. (en revision). Spanish validation of the Sexual Addiction Screening Test (SAST).

Courtice, E. L., \& Shaughnessy, K. (2018). The Partner Context of Sexual Minority Women's and Men's Cybersex Experiences: Implications for the Traditional Sexual Script. Sex Roles, 78(3-4), 272-285. https://doi.org/10.1007/s11199-017-0792-5

Daneback, K., Cooper, A., \& Mansson, S. A. (2005). An Internet study of cybersex participants. Archives of Sexual Behavior, 34, 321-328. 
Daneback, K., Sevcikova, A., Mänsson, S.A., \& Ross, M. W. (2013). Outcomes of using the internet for sexual purposes: fulfilment of sexual desires. Sexual Health, 10(1), 26-31. https://doi.org/10.1071/SH1102

Delmonico, D. (1997). Internet Sex Screening Test. Disponible en http://www.sexhelp.com el 19 de marzo de 2010

Kalichman, S. \& Rompa, D. (1995). Sexual sensation seeking and sexual compulsivity scales: reliability, validity and predicting HIV risk behavior. Journal of Personality Assessment, 65, 586-601.

Reid, R. C., Garos, S., \& Carpenter, B. N. (2011). Reliability, Validity, and Psychometric Development of the Hypersexual Behavior Inventory in an Outpatient Sample of Men. Sexual Addiction \& Compulsivity, 18(1), 30-51. http://doi.org/10.1080/10720162.2011.555709.

Salmeron, P., Ballester, R., Gómez, S., \& Gil, M.D. (2009). Adicción a internet en jóvenes. XVIII Reunión de la SPCV. Genética y ambiente: de los endofenotipos a la intervención familiar, Peñíscola, 29 y 30 de Mayo de 2009.

Shaughnessy, K., Byers, E. S., \& Walsh, L. (2011). Online sexual activity experience of heterosexual students: Gender similarities and differences. Archives of Sexual Behavior, 40, 419-427.

Wéry, A., \& Billieux, J. (2017). Problematic cybersex: Conceptualization, assessment, and treatment. Addictive Behaviors, 64, 238-246. https://doi.org/10.1016/j.addbeh.2015.11.007

Young, K. (2003). Cybersexual addiction quiz. Disponible en http://www.netaddiction.com el 19 de marzo de 2010. 
